\title{
Potential use of multiple surveillance data in the forecast of hospital admissions
}

\author{
Eric H.Y. Lau*1, Dennis K.M. Ip ${ }^{1}$ and Benjamin J. Cowling ${ }^{1}$ \\ ${ }^{1}$ School of Public Health, The University of Hong Kong, Hong Kong
}

\section{Objective}

This paper describes the potential use of multiple influenza surveillance data to forecast hospital admissions for respiratory diseases.

\section{Introduction}

A sudden surge in hospital admissions in public hospital during influenza peak season has been a challenge to healthcare and manpower planning. In Hong Kong, the timing of influenza peak seasons are variable and early short-term indication of possible surge may facilitate preparedness which could be translated into strategies such as early discharge or reallocation of extra hospital beds. In this study we explore the potential use of multiple routinely collected syndromic data in the forecast of hospital admissions.

\section{Methods}

A multivariate dynamic linear time series model was fitted to multiple syndromic data including influenza-like illness (ILI) rates among networks of public and private general practitioners (GP), and school absenteeism rates, plus drop-in fever count data from designated flu clinics (DFC) that were created during the pandemic. The latent process derived from the model has been used as a measure of the influenza activity [1]. We compare the cross-correlations between estimated influenza level based on multiple surveillance data and GP ILI data, versus accident and emergency hospital admissions with principal diagnoses of respiratory diseases and pneumonia $\&$ influenza (P\&I).

\section{Results}

The estimated influenza activity has higher cross-correlation with respiratory and P\&I admissions ( $\rho=0.66$ and 0.73 respectively) compared to that of GP ILI rates (Table 1). Cross correlations drop distinctly after lag 2 for both estimated influenza activity and GP ILI rates.

\section{Conclusions}

The use of a multivariate method to integrate information from multiple sources of influenza surveillance data may have the potential to improve forecasting of admission surge of respiratory diseases.

Table 1. Cross correlations between the estimated influenza activity based on the multivariate dynamic linear model, GP ILI rate versus A\&E respiratory diseases and P\&I admissions

\begin{tabular}{|c|c|c|c|c|c|}
\hline Surveillance data & & & Cross correlations / lags* & & \\
\hline & -2 & -1 & 0 & 1 & 2 \\
\hline Respiratory disease admissions & & & & & \\
\hline GP ILI & 0.48 & 0.53 & 0.55 & 0.50 & 0.45 \\
\hline Estimated influenza activity & 0.59 & 0.66 & 0.66 & 0.58 & 0.46 \\
\hline P\&I admissions & & & & & \\
\hline GP ILI & 0.57 & 0.62 & 0.65 & 0.59 & 0.49 \\
\hline Estimated influenza activity & 0.66 & 0.73 & 0.73 & 0.65 & 0.53 \\
\hline
\end{tabular}

*negative lags refer to correlations between lagged surveillance data and hospital admissions

\section{Keywords}

influenza; surveillance; admission; respiratory

\section{References}

Lau EH, Cheng CK, Ip DK, Cowling BJ. Situational awareness of influenza activity based on multiple streams of surveillance data using multivariate dynamic linear model. PLoS ONE 7(5): e38346. doi:10.1371/journal.pone. 0038346

*Eric H. Lau

E-mail: ehylau@hku.hk 